\title{
INTERACTION BETWEEN A REACTIVE PREFORM AND THE SURROUNDING GAS-PHASE DURING CVI
}

\author{
Gerard L. Vignoles, Cédric Descamps and Nicolas Reuge
}

August 31, 1999

\begin{abstract}
An integrated modelling approach of Chemical Vapour Infiltration of a fibrous preform by methyltrichlorosilane, featuring coupled reactor fluid mechanics and porous medium gas transport has been developed. The coupling between transport/reaction phenomena inside and outside the preform is rather strong, both physically and numerically. An important counter-effect of the reactive preform on the surrounding gas-phase composition is evidenced; also, an appreciable dissymmetry of the densification rate inside the preform, even with negligible convective transport inside it . Such an approach brings new informations for understanding the physico-chemical phenomena of CVI and new guidelines for preform densification optimisation.
\end{abstract}

\section{$1 \quad$ Introduction.}

Since many years, Chemical Vapour Infiltration (CVI) has been extensively studied, both theoretically and numerically, in order to optimise densification rates and deposit uniformity within the preform. Most of these studies dealt with the interior of the porous preform, the physico-chemical parameters outside the preform being treated as uniform and constant Dirichlet boundary conditions [1] [2] [3]. In this work, we try to address the impact of such a simplification. The first part of the present paper is a brief presentation of the whole physico-chemical problem. Then, a consistent numerical strategy is presented for its resolution. The third part is devoted to the study of a model case, where the interaction between free-medium gas transport and porous-medium reactivity is clearly illustrated.

\section{Problem statement}

\subsection{Physico-chemical phenomena}

The Chemical Vapour Infiltration process relies on the interplay between chemical species transport and chemistry, inside and outside the porous medium. Accordingly, the physico-chemical phenomena that are to be taken into account in a model are:

- Gas convection in the furnace cavity. This implies to solve also a thermal modeling problem as soon as the system is not strictly isothermal.

- Gas diffusion in the furnace cavity

- Homogeneous gas reactions

- Gas transport in a porous medium, featuring diffusion, viscous transport and Knudsen (ballistic) diffusion

- Heterogeneous (gas-solid) reactions

- Porous medium structural evolution 
This last item usually displays a much slower evolution than the preceding ones. Accordingly, an usual assumption is to treat all gas-related phenomena (transport and reactions) as in a quasi-steady state; the porous medium properties may be updated from the knowledge of this quasi-steady state.

In the following sections, we will recall briefly the equations suited to describe all these phenomena. The porous medium and the free medium will be presented separately ; the question of coupling them together will be then addressed, and finally the slower evolution of the porous medium properties.

\subsection{Gas transport and reaction in the porous medium}

The CVI equations, based on diffusion-reaction equations, have been well described in many past works. We may refer e.g. to [1] and [4] for extended descriptions.

Each species satisfies a mass conservation equation, which is a local balance between convection, diffusion, and reaction :

$$
\nabla \cdot\left(\vec{J}_{i}^{V}+\vec{J}_{i}^{D}\right)=\epsilon \sum_{l=1}^{N_{h o m}}\left(v_{i l} k_{l} \prod_{j} \frac{P_{j}^{v_{l i}}}{\mathcal{R} T}\right)+\sigma_{\imath} \sum_{l=1}^{N_{h e t}}\left(v_{i l} k_{l} \prod_{j} \frac{P_{j}^{v_{l j}}}{\mathcal{R} T}\right)
$$

The gas transport fluxes, featuring ordinary diffusion, viscous flow (from Darcy's law), and Knudsen diffusion, are evaluated using the Dusty-Gas Model [5]:

$$
\begin{gathered}
\vec{J}_{i}^{V}=-\frac{P_{i} B}{\mu \mathcal{R} T} \nabla P \\
\sum_{j \neq i}^{n} \frac{x_{i} \vec{J}_{j}^{D}-x_{j} \vec{J}_{i}^{D}}{D_{i j}}-\frac{\vec{J}_{i}^{D}}{D_{i}^{K}}=\frac{\nabla P_{i}}{\mathcal{R} T}
\end{gathered}
$$

The ordinary diffusion coefficients are given as effective quantities in the porous medium[5] derived from the gas-pair coefficients [15]:

$$
D_{i j}=\epsilon \eta_{b}^{-1} \mathcal{D}_{i j} \frac{3}{16} \frac{\sqrt{4 \pi R T}}{n \pi \sigma_{i j}^{2} \Omega_{i j}^{D}} \cdot \sqrt{2 \frac{M_{i}+M_{j}}{M_{i} M_{j}}}
$$

The Knudsen diffusion coefficients are computed the following way [5]:

$$
D_{i}^{K}=\epsilon \eta_{K}^{-1} \frac{1}{3} \sqrt{\frac{8 \mathcal{R} T}{\pi M_{i}}} d_{p}
$$

The permeability factor is :

$$
B=\epsilon \eta_{v}^{-1} \frac{d_{p}^{2}}{32}
$$

Here $d_{p}$ stands for the mean pore diameter, which may be approximated by $4 \epsilon / \sigma_{v}$. The three kinds of transport coefficients include specific geometric factors from the precise porous medium details, termed as tortuosities $\eta_{b}, \eta_{K}$, and $\eta_{v}$. There is no reason for them to be equal, except in trivial cases. Their usual range is between 1 and 10 , growing higher when close to percolation thresholds. If the medium is anisotropic, then their inverses have to be considered as tensors.

The unknowns of eqs. $(2,3)$ are the gas partial pressures since they arise quite naturally from linear thermodynamics.

Heat transfer is not treated since we are dealing with I-CVI, and thus consider the preform temperature to be constant. 


\subsection{Free Medium}

We consider in this part a classical steady-state hot-wall CVD problem, where balance equations are set for mass, momentum, heat, and species. These equations are listed below. Note that the species balance equation is formulated with the partial pressures as unknowns, so as to be consistent with the porous medium formulation.

- Momentum conservation (Navier-Stokes)

$$
\begin{gathered}
\nabla \cdot(\rho \vec{v} \vec{v})+\nabla \cdot \tau-\nabla \cdot(p I)=\sum_{k=1}^{n} \rho_{k} \vec{F}_{k} \\
\tau=-\mu\left(\nabla \vec{v}+{ }^{t} \nabla \vec{v}\right)+\frac{2}{3} \nabla \cdot \vec{v}
\end{gathered}
$$

- Mass conservation (Continuity)

$$
\nabla \cdot(\rho \vec{v})=0
$$

- Energy (Heat equation)

$$
\rho C_{p} \vec{v} \nabla T-\nabla \cdot(\lambda \nabla T)
$$

- Species conservation

$$
\nabla \cdot\left(c_{i} \vec{v}+\vec{J}_{i}^{D}\right)=\epsilon \sum_{l=1}^{N_{h \circ m}}\left(v_{i l} k_{l} \prod_{j} \frac{P_{j}^{v_{l j}}}{\mathcal{R} T}\right)
$$

The diffusive fluxes $J_{i}^{D}$ are calculated according to Stefan-Maxwell laws [16] :

$$
\sum_{j \neq i}^{n} \frac{x_{i} \vec{J}_{j}^{D}-x_{j} \vec{J}_{i}^{D}}{D_{i j}}=\frac{\nabla P_{i}}{\mathcal{R} T}
$$

Since diffusive fluxes are chosen such as they sum up to zero, and that all mole fractions sum up to one, this last relation is non-invertible. However, an approximate expression yielding explicitly the fluxes as functions of the mole fractions and their gradients is available: it is the so-called "Bifurcation method" [14] which has been used in our case.

\subsection{Interface relationships}

Coupling both media means to provide relationships for fluxes, velocities and partial pressures from one side to the other. First of all, the partial pressures have to be the same on both sides :

$$
P_{i}^{\text {porous }}=P_{i}^{\text {free }}
$$

This is so because the porous medium partial pressures are defined as fluid-phase average quantities and not global average quantities.

Concerning fluxes, some confusion should be avoided on Knudsen diffusion. Indeed, even if it has a formally diffusive character (although having a microscopically radiative nature), this phenomenon also displaces the barycenter of the fluid, which is usually considered as convection, more precisely, as "separative convection" in this case. One consequence of such a fact is that the Dusty-Gas Model fluxes do not sum up to zero (however, there exists a relationship between them, known as Graham's law), so they are not to be considered as "purely diffusive", while the viscous fluxes do not represent the whole of the convection. Consequently, it is not possible to equate separately the diffusive fluxes and the viscous fluxes from both sides. The correct relations are equalities of the total fluxes:

$$
\vec{J}_{i}^{\text {porous }}=\vec{J}_{i}^{\text {free }}=C_{i} \vec{v}^{\text {free }}+\vec{J}_{i}^{D, \text { free }}
$$


Since the free-medium velocity appears directly here, we have then to write the following extra interface relationship between the summed DGM mass fluxes on one side and the barycentric velocity on the other :

$$
\sum_{i=1}^{N} M_{i} \vec{J}_{i}^{\text {porous }}=\rho \vec{v}^{\text {free }}
$$

A heterogeneous deposition reaction may also occur at the interface, more precisely at its the solid fraction surface. Accordingly, the free-medium fluxes should be equated to the sum of the porous-medium fluxes and of the chemical interface deposition flux :

$$
R_{i}=\epsilon \sum_{l=1}^{N_{\text {het }}}\left(v_{i l} k_{l} \prod_{j} \frac{P_{j}^{v_{l_{i}}}}{\mathcal{R} T}\right)
$$

However, this reactive flux is usually unimportant with respect to the porous medium flux.

\subsection{Structural Evolution}

Once a stationary state has been reached with the former procedure, time integration is performed, in order to take into account the evolution of the structural parameters of the porous preform. For this, it is assumed that this evolution is very slow compared to all other variables related to the gas. Accordingly, explicit integration may be performed.

We follow only one parameter, that is, the porosity $\epsilon$, which is known to decrease continuously with time in such a process. It is updated according to the following formula :

$$
\frac{\partial \epsilon}{\partial t}=\sigma_{\nu}(\epsilon) \sum_{i}^{N_{d e p}}\left(V_{m i} k_{i} \prod_{j} C_{j}^{v_{i j}}\right)
$$

This equation relies formally on two structural parameters, the porosity $\epsilon$ and the volumic surface $\sigma_{v}$. However, the latter may be considered as a function of the former. In classical cases of porous media geometries, analytical or fitted laws do exist [6]. Otherwise, the dependence $\sigma_{v}=f(\epsilon)$ is attainable through homogenization approaches [7]. In both cases, it is then possible to treat eq. 17 as an ODE for $\epsilon$ only, which may be solved using a classical Runge-Kutta algorithm.

Before going back to the determination of a new fluid mechanical steady state, all properties depending on $\epsilon$ are updated. These are $: \sigma_{v}, d_{h}=\frac{4 \epsilon}{\sigma_{v}}$, and the tortuosity factors for the three kinds of transport involved in the Dusty-Gas Model, namely $\eta_{v}, \eta_{b}$, and $\eta_{K}$. All these parameters may be made dependent on $\epsilon$ alone as for $\sigma_{v}$.

\section{Coupling Both Media : Numerical Procedure}

\subsection{Principle}

At first sight, the free-medium and porous-medium formulations are very similar, since they are balance equations featuring convective and diffusive fluxes, as well as chemical sources or sinks. Indeed, in a preceding work [8], all of them had been solved simultaneously inside and ouside the preform ; decoupling between heat, mass and momentum balances on one part and species balances on the other part had been performed in order to reduce computational effort. This approach failed indeed to converge numerically in cases of low permeability values. The reason for such a problem lies in the fact that Knudsen diffusion has also a convective character which strongly couples the continuity equation along with the species balance equations inside the porous preform only.

A first attempt to circumvent this drawback has been to remove all uncoupling, that is, to solve simultaneously all equations on the whole domain. The results were not satisfactory : numerical oscillations arised close to the preform surface. They were also due to the difference in balance equations couplings between the two kinds of medium.

To avoid such a numerical behaviour, some underrelaxation has to be introduced at the interface. Accordingly, 
a new decoupled scheme has been set up, where free-medium and porous medium problems are alternatively solved. The chosen algorithm was the following one :

- 1. First, solve all balance equations in the free-medium, considering fixed-flux B.C.'s at the preform surface.

- 2. Extract the computed partial pressures at the surface and consider them as fixed Dirichlet conditions for the porous-medium resolution.

- 3. Extract the computed total fluxes at the preform surface and treat them as non-homogeneous Neumann B.C.'s for a new free-medium resolution.

- 4. Repeat steps 2 and 3 until convergence.

At each B.C. extraction (either Dirichlet or Neumann), underrelaxation is performed by blending together results from steps $n$ and $n-1$.

The choice of a starting point as close as possible to the final solution accelerates appreciably the convergence. To do so, we compute a first guess for the diffusive fluxes from the solution of a 1D simplified diffusion-reaction problem. For example, in the case of a $1^{s t}$-order consumption reaction of species $i$, we have :

$$
J_{i}^{D}=-\frac{\epsilon D_{p o r} \Phi \tanh (\Phi)}{L} \frac{P_{i}}{R T}
$$

where $\Phi$ is the Thiele modulus :

$$
\Phi=L \sqrt{\frac{k \sigma_{v}}{\epsilon D_{p o r}}}
$$

The first step of this algorithm (free-medium balance equations) has been split into two substeps :

- Resolution of Navier-Stokes and continuity equations for a given mean molar mass ;

- Resolution of mass-balance equations for the $n$ chemical species for a given velocity field.

The two substeps are iteratively performed until convergence, that is, until global mass and mole conservations are effective.

The mass-balance equations had to be solved using an adaptation of a numerical procedure due to Giovangigli [13]. Indeed, the singular nature of the Stefan-Maxwell fluxes, even partially removed by the "bifurcation" approximation scheme, is a severe drawback for numerical convergence. An artificial extra term allowing each species to diffuse down the gradient of the total pressure (which has to be close to zero in laminar flows) ensures rapid and accurate results because it prevents any total pressure gradient buildup to occur during resolution.

\subsection{Underrelaxation}

We show here from what the necessity of underrelaxation arises, using a very simplified case [12]: 1D geometry, symmetry axis inside the preform, diffusion of one single species.

Consider that diffusion and convection in the free-medium yield a diffusive boundary layer of thickness $\delta$ around the preform. Then a relation may be written between the interface concentration $C_{i}$, the bulk-gas concentration $C_{b}$, and the interface flux $J$ :

$$
-D_{f r e e} \frac{C_{b}-C_{I}}{\delta}=J
$$

In the porous medium of thickness $2 L$, eqns. 18 and 19 give another expression for the flux as a function of $C_{I}$ as soon as Darcy flow is assumed negligible. Interchanging Neumann (fixed flux) and Dirichlet (fixed concentration) B.C.'s is then equivalent to a dynamical iterative scheme :

$$
f: C_{I}^{n} \rightarrow C_{I}^{n+1}=C_{b}-\frac{\delta}{L} \frac{\epsilon D_{\text {por }}}{D_{\text {free }}} \Phi \tanh (\Phi) \cdot C_{I}^{n}=C_{b}-p \cdot C_{I}^{n}
$$


From elementary theory of iterated suites, this scheme converges if and only if $p=\left|\frac{\partial C_{I}^{n+1}}{\partial C_{I}^{n}}\right|<1$. This is physically achieved when :

- The Thiele modulus is low : slow kinetics, as compared to diffusion (usually at low temperatures)

- The ratio of convection to diffusion is high in the free medium.

- The Knudsen number $K n=\frac{D_{\text {free }}}{D_{\text {por }}}$ is high, which means low pressure, high temperature, or small pore diameter.

By the way, these three cases correspond to highly unefficient CVI conditions. On a numerical point of view, as soon as the estimated value of $p$ approaches 1 , then underrelaxation has to be performed, for instance on the concentrations :

$$
C_{I}^{n+1}=\alpha C_{I}^{n}+(1-\alpha) f\left(C_{I}^{n}\right)
$$

An optimal lower bound for $\alpha$ would be then $\frac{p-1}{p+1}$.

\section{$4 \quad$ A Model Case Study}

\subsection{Case Definition}

In order to evaluate the procedure, we have chosen a rather simple and classical case [8]. From a chemical point of view, only one heterogeneous reaction is involved, in which SiC is deposited from methyltrichlorosilane (MTS) :

$$
\mathrm{CH}_{3} \mathrm{SiCl}_{3} \rightarrow \mathrm{SiC}_{(s)}+3 \mathrm{HCl}
$$

The main feature of such a reaction is that mass decrease and mole number increase occur simultaneously for the gas phase. The reaction deposition constant has been taken from [9] :

$$
k_{M T S}\left(m . s^{-1}\right)=3.89 .10^{9} \exp \left(-\frac{296.10^{3}}{\mathcal{R} T}\right)
$$

The considered chemical species are three : $M T S, H C l$, and a dilution agent, $\mathrm{H}_{2}$. Considering that no homogeneous reaction occurs in the process is an oversimplification which may lead to rather unphysical results ; however, it is enough to reveal how porous medium and free medium interact.

The considered geometry is a cylindrical preform located inside a 5-cm radius tubular furnace. A thermal profile inspired from [11] has been assigned to the walls and the preform (2cm radius and $4 \mathrm{~cm}$ height) is held at a constant temperature of $1200 \mathrm{~K}$.

A consistent set of structural parameters laws has been taken considering the case of an isotropic random packing of fully overlapping capillaries: these laws have been reported in [10]. The porous medium properties were prior to densification : $\epsilon=0.8, r_{f}=7 \mu m, \sigma_{v}=91900 m^{-1}$, which yields at $1200 \mathrm{~K}$ a Thiele modulus of about 2 , and a Knudsen number of 5 . This situation is interesting in that all phenomena are in competition, except Darcy flow that is small with respect to diffusion (the ratio is around $10^{-2}$ ). Isotropy in the transport properties is preserved when this last condition is verified.

\subsection{Numerical convergence}

A rapid evaluation of parameter $p$ involved in the preceding section yields values ranging from 1 to 3 , depending on the inlet mass flow rate ; thus, underrelaxation has been performed with a factor $\alpha=0.5$. As a matter of fact, underrelaxation had also to be made between the two free-medium substeps. The lengthiest part of the computation is related to the determination of all fields at the initial steady state, since it has to be reached through a double loop. 


\subsection{Results and discussion}

The MTS field exhibits four zones, as illustrated at figure 1 :

- i) A convection-dominated zone at the inlet of the furnace,

- ii) a slightly marked diffusive boundary layer around the preform,

- iii) another convection-dominated zone at the outlet of the furnace, and

- iv) a depletion zone inside the preform.

Note that zone ii) has a smoother contour than the preform itself, resulting in a higher partial pressure at the preform corners. The partial pressure drop inside zone ii) (which is here only due to diffusion) is appreciable. This validates the pertinence of a coupled simulation as compared to a simple Dirichlet B.C. statement. Also, the depletion zone iv) exhibits a maximal depletion point somewhat shifted towards the outlet of the preform, even if the fluxes are essentially diffusive inside the preform.

Figure 2 displays the partial pressure fields in the whole resolution space at the initial time. $H C l$ displays an important counterflow-diffusion effect towards the furnace inlet. On the other hand, $P_{H_{2}}$ decreases slightly towards the outlet, essentially because of the gas acceleration. The alteration of $P_{H_{2}}$ around the preform relies only on multicomponent diffusion.

Fig. 3 displays the evolution of the porosity field inside the preform as the process goes on ; it is easily seen how the least-density point goes back towards the center of the preform.

\section{Conclusion}

A comprehensive numerical approach for the modelling of CVI has been developed, including porous-medium and free-medium transport, as well as interface exchanges. The coupling between both media is strong, which implies that underrelaxation is required in order to get a satisfactory numerical solution. Also, precursor depletion effects close to the preform may be safely accounted for, instead of more traditional fixed-concentration B.C's. The study of a model case where a cylindrical preform is held inside a tubular furnace showed that the spatial repartition of gases inside the preform depends somewhat on the external gas flow pattern because of a downstream depletion effect, even if the convective flux is negligible inside it.

With such a modelling tool in hand, many special effects may now be investigated, like the interaction between adjacent preforms, the effect of preform anisotropy on free-medium gas circulation, etc...

\section{Acknowledgements}

This work was supported by SEP, division of SNECMA through PhD grants to C.D. and N.R.

Computations were carried out on the commercial code FLUX-EXPERT, distributed by Simulog France. Software and hardware purchase has been made possible thanks to SEP and Conseil Régional d' Aquitaine.

The authors wish to acknowledge V. Giovangigli (Ecole Polytechnique, Palaiseau, France), M. Pons (LTPCM Grenoble, France), and S.V. Sotirchos (University of Rochester, NY, USA) for helpful discussions. 


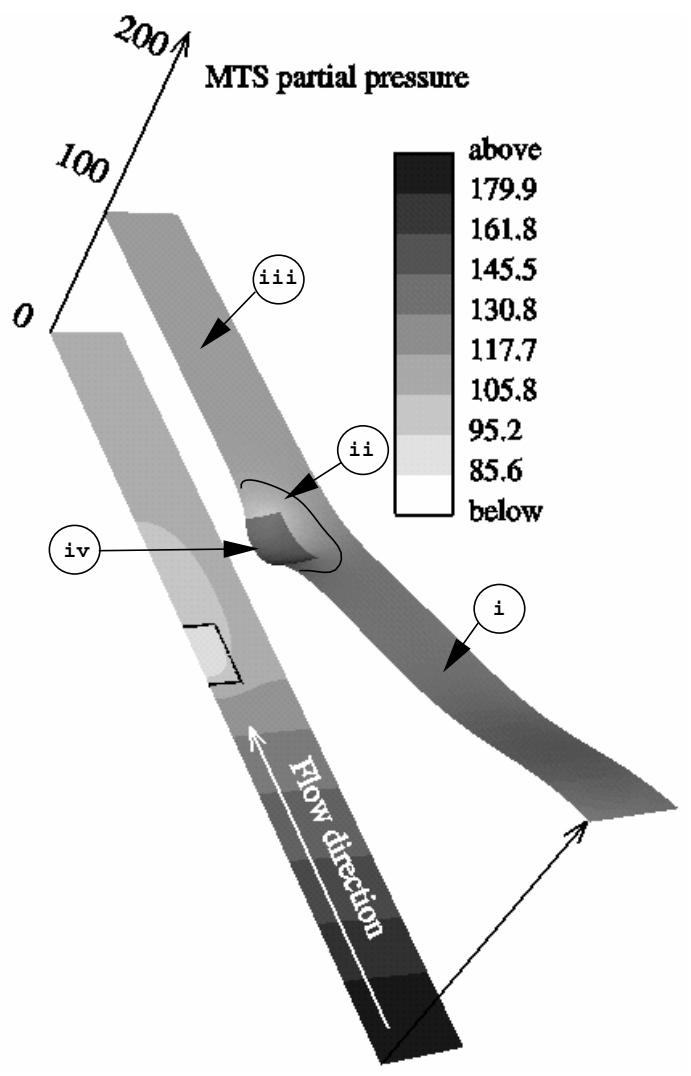

Figure 1 : Elevation view of the MTS partial pressure in the furnace and the preform at the beginning of the process



Figure 2 : Partial pressure fields for the three gaseous species at initial densification stage Left:MTS Middle: $\mathrm{HCl}$ Right: $\mathrm{H}_{2}$ 
Porosity

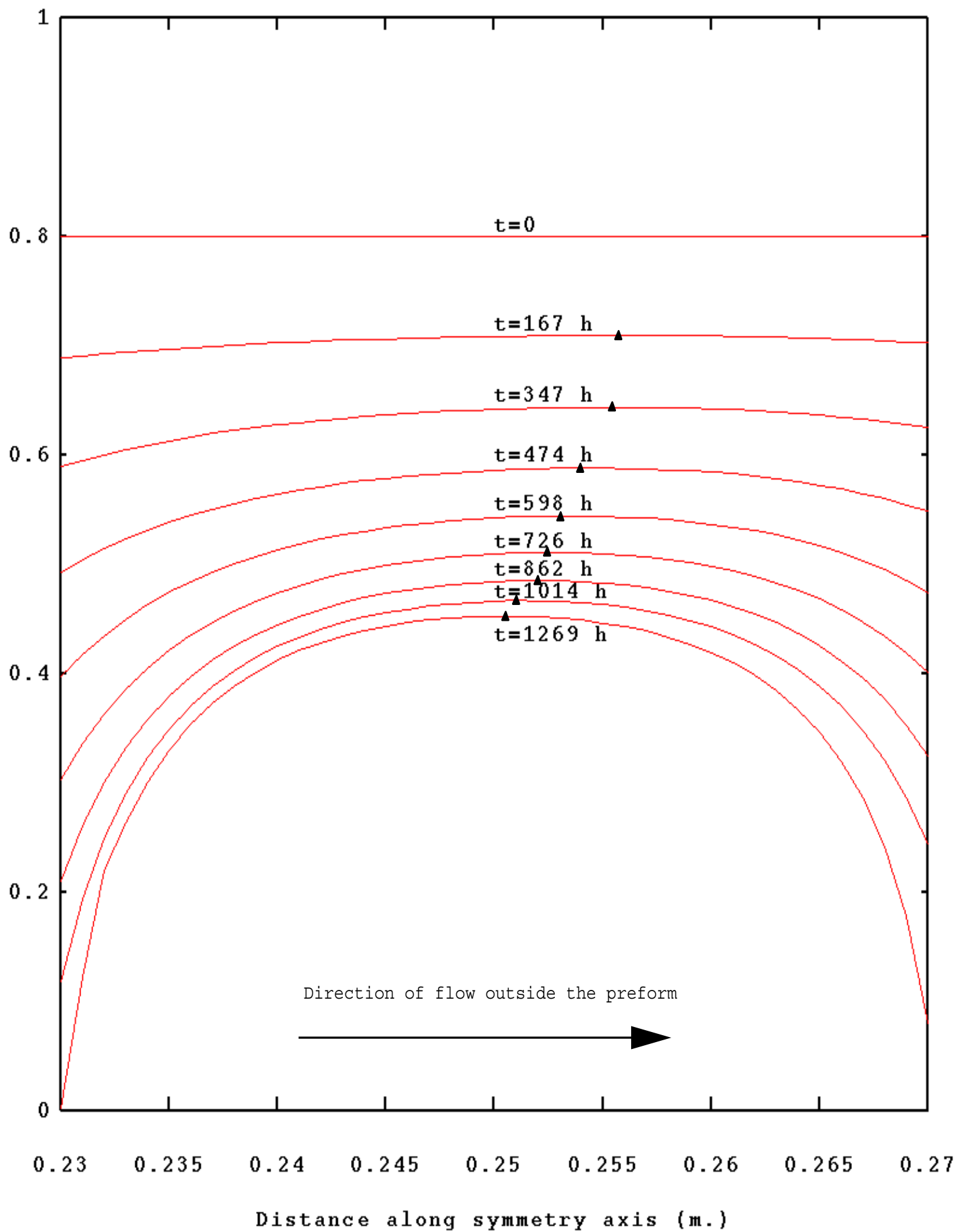

Figure 3 : Time evolution of the porosity in the preform along the symmetry axis.

The plain triangles mark the least-density points. 


\section{References}

[1] Dynamic Modelling of Chemical Vapor Infiltration, S.V. Sotirchos, AIChE J. 37, 1365 (1991).

[2] Optimum Conditions for Composites Fiber Coating by Chemical Vapor Deposition, S.K. Griffiths and R.H. Nilson, J. Electrochem. Soc. 145(4), 1263-1272 (1998)

[3] ,Chemical Vapor Infiltration - Modelling Solid Matrix Deposition in Ceramic-Matrix Composites, G.Y. Chung, B.J. McCoy and J.M. Smith, Chem. Eng. Sci 46(3),723-734 (1991)

[4] The interaction of chemical kinetics and diffusion in the dynamics of chemical vapor infiltration, S. Middleman, J. Mater. Res. 4(6), 1515 (1989)

[5] Gas Transport in Porous Media: The Dusty-Gas Model, E.A. Mason and M.M. Malinauskas, Chemical Engineering Monographs 17, Elsevier, Amsterdam (1983).

[6] Effective Knudsen diffusivities in structures of randomly overlapping fibers, M.M. Tomadakis and S.V. Sotirchos, AIChE J. 37(1), 74-86 (1991)

[7] Numerical study of the coupled evolution of micro-geometry and transport properties of simple 3D porous media, D. Bernard, G.L. Vignoles, in "Computational Methods for Flow and Transport in Porous Media", J.-M. Crole ed., Kluwer Acad. Pub., Dordrecht (1999).

[8] Finite Element Modelling of Chemical Vapor Infiltration, G.L. Vignoles and C. Descamps, Euro-CVD 11/ICVD 14, Paris, September 1997.

[9] G. Schoch, W. Fritz and E. Fitzer, Tech. Rep. EURAM Contract MAIE/0018/C (1991)

[10] Structural Model Effects on the Predictions of Chemical Vapor Infiltration Models, J.Y. Ofori and S.V. Sotirchos, J. Electrochem. Soc. 143(6), 1962-1973 (1996)

[11] O.Feron, PhD Thesis no. 1867, University of Bordeaux 1 (1998)

[12] Interaction between a reactive preform and the surrounding gas-phase during CVI, G.L. Vignoles and C. Descamps, Modelling of Reaction Fronts Workshop, April 19-21 1999, Lyon, France.

[13] Mass Conservation and Singular Multicomponent Diffusion Algorithms, V. Giovangigli, Impact of Computing in Science and Engineering 2, 73-97 (1990).

[14] An analysis of the coupled chemically reacting boundary layer and charring ablator. Part IV : A unified approximation for mixture transport properties for multicomponent boundary layer applications, E.P. Bartlett, R.M. Kendall, R.A. Rindal, NASA report CR-1063 (1967).

[15] The properties of gases and liquids, R.C. Reid, J.M. Prausnitz, B. E. Poling, Mc Graw Hill Book Company, New York (1987).

[16] Transport Phenomena, R.B. Bird, W.E. Stewart and E.N. Lightfoot, John Wiley and Sons, New York (1960) 